\title{
Electromagnetic signals related to incidence of a teleseismic body wave into a subsurface piezoelectric body
}

\author{
Tsutomu Ogawa and Hisashi Utada \\ Earthquake Research Institute, University of Tokyo, Tokyo 113-0032, Japan \\ (Received June 18, 1999; Revised February 2, 2000; Accepted March 6, 2000)
}

\begin{abstract}
This paper presents a case study of Electromagnetic (EM) signals associated with earthquakes due to the piezoelectricity of crustal rocks. For a simple model of crustal structure with a subsurface piezoelectric body, a mathematical expression was obtained that describes the behavior of piezoelectric EM signals due to incidence of a teleseismic body wave. Using this expression, we evaluated expected EM signals with physical parameters reasonable for crustal rocks. Results of the frequency domain analysis suggested that the intensity of the signal decreases with decreasing frequency due to decreasing stress rate at lower frequencies, and decreases with increasing frequency due to EM attenuation in the conducting medium at higher frequencies. However, the latter (the skin effect) was shown to be negligible at the dominant frequency range of seismic waves so far as a shallower piezoelectric body is concerned. Numerical results also indicated a resonant feature of the piezoelectric EM signals corresponding to geometry of the subsurface piezoelectric body. However, numerical calculations suggested that such signals cannot be detected except for strong motions. If detected, on the other hand, their spatial and frequency characteristics will provide information on the geometry of the subsurface piezoelectric body.
\end{abstract}

\section{Introduction}

Observational studies have reported electromagnetic (EM) field variations prior, or simultaneous to earthquakes around the epicentral region (e.g., Gokhberg et al., 1982; Warwick et al., 1982; Fraser-Smith et al., 1990; Mueller and Johnston, 1990; Johnston et al., 1994). Drastic change of the stress field and related phenomena in the crust associated with earthquakes possibly induce EM phenomena.

Several models, such as piezomagnetism, the electrokinetic effect and piezoelectricity, have been proposed as possible mechanisms to generate coseismic EM signals related to earthquakes. There have been a number of works that quantitatively discussed coseismic change of the magnetic field around the epicentral region on the basis of the piezomagnetism of the rock, with surrounding coseismic stress change and the Curie depth taken into account (e.g., Sasai, 1991; Johnston et al., 1994). Although the coseismic stress field variation due to earthquakes should well be quantified in terms of seismology, coseismic EM signals on the basis of certain mechanisms other than piezomagnetism with realistic models of the space have not been well simulated. Among these generation mechanisms, this paper pays special attention to piezoelectricity that arises from anisotropy of the substance and has been well studied since its discovery in the 19th century (e.g., Ikeda, 1990).

Experiments showed that some kinds of rocks containing piezoelectric crystals have bulk piezoelectricity (Parkhomenko, 1971; Bishop, 1981b). Though Tuck et al. (1977) denied the presence of bulk piezoelectricity, recent experimental

Copy right(c) The Society of Geomagnetism and Earth, Planetary and Space Sciences (SGEPSS); The Seismological Society of Japan; The Volcanological Society of Japan; The Geodetic Society of Japan; The Japanese Society for Planetary Sciences. studies by Ghomshei et al. (1988) and Ghomshei and Templeton (1989) showed the presence of bulk piezoelectricity and a quasi-homogeneous $a$-axes fabric at the scale of a hundred meters, in which piezoelectric axes are oriented to a certain direction. Although the presence of a rock's piezoelectric signal in a laboratory scale was well confirmed by stick-slip experiments by Yoshida et al. (1994), it is still controversial whether a signal due to the same mechanism can be observed in the real earth associated with earthquakes, and whether crustal rocks show bulk piezoelectricity of geologic scale. Nevertheless, EM observations have been interpreted by this mechanism, i.e., by assuming bulk piezoelectricity in the crust (Warwick et al., 1982; Huang, 1999).

In the previous paper (Ogawa and Utada, 2000), we obtained an analytic expression for the piezoelectric signals due to a fault motion of an earthquake, and numerically evaluated the signal behaviors in a uniform whole space. One of the most important conclusions obtained by this work is that observation of such signals will reveal anisotropy of the medium at the earthquake source region, though detection of such signal will be quite difficult. On the other hands, it is claimed that crustal rocks will not be piezoelectric in general, for the observational facts that electric measurements in the granite-rich area do not record signals associated with incidence of teleseismic waves (Johnston, 1998, personal communication). Obviously, quantitative discussion is necessary to answer the question about the absence of teleseismically induced piezoelectric signals. It is because detectability depends on signal to noise ratio, i.e., comparison between expected signal intensity and both the resolution of the installed measurement system and the noise level at the observation point are indispensable. 
There have been several theoretical works for such purposes in terms of other physical processes. For example, Eleman (1965) quantitatively examined magnetic field variations related to arrival of the surface waves into a crustal rock due to its piezomagnetism. Another study by Mikhailov et al. (1997) showed a reasonable agreement between observed and calculated electric field variations, in which they presumed electrokinetic coupling as its origin. As for the prospecting of mineral piezoelectric crystals, Kepic et al. (1995) estimated the distribution of sulfide minerals by simultaneous measurement of the acoustic wave and the electric field during an explosion experiment, which indicated that in-situ electric signal detection is possible under a special condition. Russell and Barker (1991) also numerically estimated amplitude of seismo-electric signal presuming a piezoelectric sphere embedded under the ground, expected in seismo-electric exploration. It showed consistency between amplitudes of the observed and expected piezoelectric signals. However, their assumptions for estimation are too much simplified, hence it omitted some interpretations of the feature of expected signals, such as a relation between the amplitude and geometry of the piezoelectric body.

In the present paper, we examined the presence of coseismic EM signals observed on the earth, by assuming the theory of linear piezoelectricity of crustal rocks in order to answer the question raised by Johnston (1998, personal communication). For the purpose, we calculated expected EM signals due to incidence of teleseismic body wave, to obtain the relation between amplitudes of piezoelectric signal and teleseismic body wave by a numerical modeling.

\section{Model}

We suppose that the earth is a half space in which the electrical and elastic properties are uniform. The medium is piezoelectric only in the cylindrical body embedded in the conductive crust as shown in Fig. 1. The radius of the body is assumed to be $5 \mathrm{~km}$, representing the scale of Mt. Tsukuba located in the northern Kanto area, one of well-known granite lenses in Japan. Depth to its top surface and its thickness are denoted as $h_{1}$ and $h_{2}$, respectively.

We assume that the piezoelectric body has the symmetry class of $\infty$ mm of the limiting group after experimental results by Parkhomenko (1971) and Bishop (1981b). The symmetry axis is assumed to be parallel to the $z$-axis. Therefore the piezoelectric coefficients are represented by a matrix in Cartesian coordinate system as

$$
\mathbf{d}=\left[\begin{array}{cccccc}
0 & 0 & 0 & 0 & d_{15} & 0 \\
0 & 0 & 0 & d_{15} & 0 & 0 \\
d_{31} & d_{31} & d_{33} & 0 & 0 & 0
\end{array}\right],
$$

where $d_{31}=-0.5 \times d_{33}, d_{15}=-1 \times d_{33}, d_{33}=1 \times 10^{-15}$ [C/N] (Parkhomenko, 1971; Bishop, 1981a). The ratios between elements are those for a quartz aggregate which consists of equal proportions of both enantiomorphs, and the $a$ axes of the two hands are antiparallel to each other, obtained theoretically by Bishop (1981a) and adopted for experimental studies by Ghomshei et al. (1988) and Ghomshei and Templeton (1989). The absolute value of piezoelectric coefficients representing those of quartz-rich rocks in the crust,

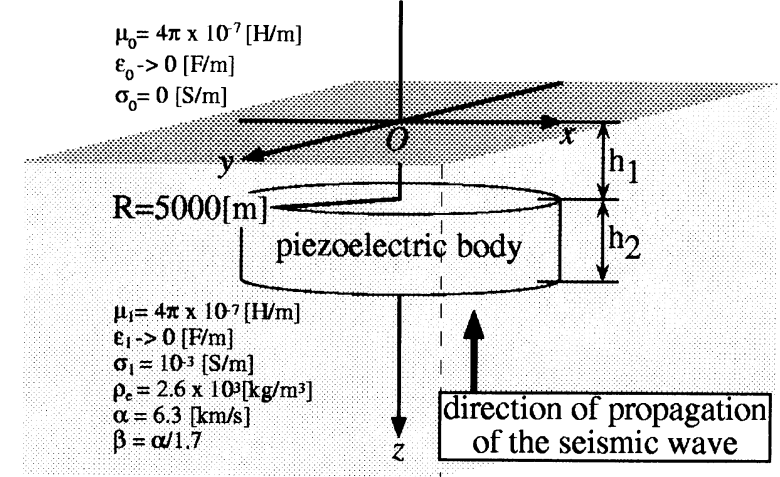

Fig. 1. Parameters substituted in this study and the shape of the piezoelectric body.

adopted and denoted above are by 3 orders of magnitude smaller than those for pure single-crystal quartz (Bishop, 1981b). The value is smaller by one order of magnitude than the value obtained by recent results of Ghomshei et al. (1988). We defined the sign of $d_{33}$ as positive when positive charge is induced in the positive direction of the $z$-axis under an extension stress in the $z$-direction.

For simplicity, the teleseismic $P$ wave is assumed to be a plane wave propagating upward parallel to the $z$-axis, i.e., incident $P$ wave is normal to the bottom of the piezoelectric body and to the earth's surface.

\section{Fundamental Equations and Solutions}

Fundamental equations are Maxwell equations for EM fields, Eulerian equation of motion for an elastic body,

$$
\begin{gathered}
\nabla \cdot \boldsymbol{D}=\rho, \quad \nabla \cdot \boldsymbol{B}=0, \\
\nabla \times \boldsymbol{E}=-\frac{\partial \boldsymbol{B}}{\partial t}, \quad \nabla \times \boldsymbol{H}=\boldsymbol{j}+\frac{\partial \boldsymbol{D}}{\partial t}, \\
\rho_{e} \frac{\partial^{2} u_{i}}{\partial t^{2}}=f_{i}+\frac{\partial \mathrm{T}_{i j}}{\partial x_{j}}
\end{gathered}
$$

and related constitutive laws expressed as

$$
\begin{gathered}
\boldsymbol{j}=\sigma \boldsymbol{E}, \\
\boldsymbol{B}=\mu \boldsymbol{H}, \\
\boldsymbol{D}=\varepsilon \boldsymbol{E}+\mathbf{d} \boldsymbol{T}, \\
\mathrm{T}_{i j}=\delta_{i j} \lambda_{e}(\nabla \cdot \boldsymbol{u})+\mu_{e}\left(\frac{\partial u_{i}}{\partial x_{j}}+\frac{\partial u_{j}}{\partial x_{i}}\right),
\end{gathered}
$$

where, $\boldsymbol{D}, \boldsymbol{B}, \boldsymbol{E}, \boldsymbol{H}, \rho, \boldsymbol{j}, \rho_{e}, u_{i}, f_{i}, \mathrm{~T}_{i j}, \sigma, \varepsilon, \mu, \mathbf{d}, \lambda_{e}, \mu_{e}$ are the electric flux density, the magnetic flux density, the electric field, the magnetic field, the volumetric electric charge density, the electric current density, the density of the medium, the $i$ 'th component of the displacement, the $i$ 'th component of the body force, the $(i j)$ element of the stress tensor, the electrical conductivity, the electric permittivity, the magnetic permeability, the piezoelectric coefficients, and the Lamé's constants, respectively (Ogawa and Utada, 2000). MKSA unit system is adopted in this study. $\boldsymbol{T}$ is a vector involving stress components defined so as to satisfy the relation,

$$
\boldsymbol{T}={ }^{t}\left(\begin{array}{llllll}
\mathrm{T}_{11} & \mathrm{~T}_{22} & \mathrm{~T}_{33} & \mathrm{~T}_{23} & \mathrm{~T}_{31} & \mathrm{~T}_{12}
\end{array}\right) .
$$


Note that one of the constitutive laws (8) is originally written as $\mathbf{S}=s \mathbf{T}+\mathbf{d} \boldsymbol{E}$, where $s$ is the compliance. However, the contribution of the electric field $\mathbf{d} \boldsymbol{E}$ in this equation can be neglected, as it is insignificant (Ogawa and Utada, 2000). The coordinate system is chosen so that the $z$-axis is downward positive and $z=0$ at the earth's surface. EM fields in the air $(z<0)$ are calculated using Green tensors for EM fields induced by an electric current element in the earth $(z>0)$. Piezoelectric EM fields are expressed as follows,

$$
\begin{aligned}
& E_{i}(\boldsymbol{r}, \omega)=\int_{V p} \sum_{j=1}^{3} \sum_{k=1}^{6} G_{i j}^{E}\left(\boldsymbol{r} \mid \boldsymbol{r}^{\prime} ; \omega\right) \\
& \times d_{j k} i \omega T_{k}\left(\boldsymbol{r}^{\prime}, \omega\right) d V^{\prime}, \\
& B_{i}(\boldsymbol{r}, \omega)=\int_{V p} \sum_{j=1}^{3} \sum_{k=1}^{6} G_{i j}^{B}\left(\boldsymbol{r} \mid \boldsymbol{r}^{\prime} ; \omega\right) \\
& \times d_{j k} i \omega T_{k}\left(\boldsymbol{r}^{\prime}, \omega\right) d V^{\prime},
\end{aligned}
$$

where $V p$ is a volumetric domain of the piezoelectric body. $G_{i j}^{E}$ and $G_{i j}^{B}$ are Green tensors of total EM fields for a uniform half space derived by Stoyer (1977) and shown in Appendix. In this study, we solve the piezoelectric EM fields observed on the earth's surface before the elastic wave reaches the surface. Therefore, the whole space expression of the stress field induced by propagation of the elastic wave is applied using the scalar potential of the displacement (Aki and Richards, 1980). Thus $T_{k}$ can be derived using relationships (8), (9) and the expression of the displacement, $\boldsymbol{u}$ due to the incident $P$ wave in the whole space,

$$
\boldsymbol{u}\left(\boldsymbol{r}^{\prime}, t^{\prime}\right)=\nabla^{\prime} \Phi\left(\boldsymbol{r}^{\prime}, t^{\prime}\right)=\boldsymbol{u}_{0} e^{i \omega(z+\alpha t)},
$$

where $\nabla^{\prime}={ }^{t}\left(\partial / \partial x^{\prime}, \partial / \partial y^{\prime}, \partial / \partial z^{\prime}\right)$ and $\Phi$ is the scaler potential for the displacement. Using analytic expressions of Green functions for the EM fields and the elastic wave, expressions of the EM fields generated from the entire piezoelectric body can be obtained by their volumetric integration over the body. Since the elastic wave is much slower than light, the displacement current, the term $\varepsilon \partial \boldsymbol{E} / \partial t$ appearing by substitution of (7) into (3), was ignored, which corresponds to letting the velocity of the speed of light be infinite $(\varepsilon \rightarrow 0)$. Expected piezoelectric EM signals are numerically evaluated with these analytic expressions, by assuming proper values of physical parameters for crustal materials (Fig. 1). According to the axisymmetry of the space and the incident plane $P$ wave, the volumetric integration in (10) and (11) was carried out analytically. We substituted parameters for elastic properties of the lower half space acceptable for those of the upper crust in the northern Kanto area, Japan (Yoshii, 1979). The Hankel transforms appearing in expressions for piezoelectric EM fields are numerically calculated using the FHT (Fast Hankel Transform) algorithm by Anderson (1982).

\section{Results and Discussion}

The calculated electric field observed on the earth's surface $2 \mathrm{~km}$ away from the origin along the $x$-axis is shown hereafter. The magnetic field does not exist in the air, due to symmetry of the piezoelectric body and the space. In other words, the present model generates only the toroidal magnetic mode.
First, absolute values of complex Fourier amplitude of the electric field are shown, for incident $P$ wave of unit velocity $1[\mathrm{~m} /(\mathrm{s} \mathrm{Hz})]$ on the earth's surface, for the case $h_{1}=100 \mathrm{~m}$ and $h_{2}=5 \mathrm{~km}$.

The electric field intensity in the lower frequency range tends to decrease with decreasing frequency due to decreasing stress rate. At higher frequencies, it decreases with increasing frequency due to EM attenuation in the conducting medium (the skin effect). It can be expected that the electric field at higher frequencies attenuates more heavily as the top of the piezoelectric body lies deeper. Vertical and horizontal components are both maximized around $1[\mathrm{~Hz}]$ and amount to about $1[\mu \mathrm{V} / \mathrm{m} /(\mathrm{m} /(\mathrm{s} \mathrm{Hz})) / \mathrm{Hz}]$. Since the amplitude of the velocity of teleseismic body wave is supposed to be several $[\mathrm{cm} / \mathrm{s}]$ at most, the electric field on the surface will not easily exceed the noise level of measurement systems, even if magnitude of the piezoelectric coefficients are 1 order larger than those adopted in the present study.

Figure 2 suggests the existence of a resonant feature of the electric field. To examine this feature, we compared absolute values of complex Fourier amplitude of $E_{z}$ for $h_{2}=5 \mathrm{~km}$ and those for $h_{2}=2.5 \mathrm{~km}$, as shown in Fig. 3. The result suggests that the interval of peak frequencies becomes twice as large, as $h_{2}$ becomes half, and that the amplitude of $E_{z}$ is approximately maximized for frequencies $f_{n}$ satisfying a condition

$$
\frac{v_{p}}{f_{n}}=\lambda_{n} \simeq \frac{h_{2}}{(2 n-1) / 2},
$$

where $v_{p}, \lambda_{n}$ and $n$ are the speed and the wavelength of the incident $P$ wave, and a natural number, respectively. The result suggests that the resonant feature in the electric field is produced by a resonance of the piezoelectric field coupled with $P$ wave in the cylindrical body.

In order to ascertain the skin effect, we compared results with $h_{1}=10,100,1000$ and $10000 \mathrm{~m}$, respectively, as shown in Fig. 4. It shows that, in the frequency range of the observable teleseismic wave, from 0.1 to $10 \mathrm{~Hz}$, the skin effect is less significant so that attenuation of the electric field through the conducting materials is negligible. In the frequency range of present interest, amplitudes of the electric field on the surface varies only within 1 order of magnitude even if the depth varies from $10 \mathrm{~m}$ to $10 \mathrm{~km}$. The skin effect becomes remarkable, firstly in the higher frequencies and secondly for the deeper piezoelectric body. Theoretically, the skin depths estimated for frequencies $0.1,1$ and 10 [Hz] and the conductivity $\sigma=10^{-3}[\mathrm{~S} / \mathrm{m}]$ are approximately 50 , 16 and $5[\mathrm{~km}]$, respectively. Thus the skin effect is expected to be less effective for a shallower piezoelectric body and the typical frequency range of seismic waves.

Spatial variation of Fourier amplitude of $E_{z}$ in the air is shown in Fig. 5, indicating that $E_{z}$ attenuates with increasing height. However, this does not mean that strong $E_{z}$ signal is expected in the ground. On the contrary, $E_{z}$ just below the surface is supposed to be of negligible amplitude due to the continuity condition of the electric current density.

Since the earth is composed of moderately conducting material, seismic waves will cause the electric field due to motional induction. The motionally induced EM fields can be obtained by an integration similar to (10) or (11), by replacing the piezoelectric source term to the induced current, $\sigma \boldsymbol{v} \times \boldsymbol{B}_{0}$, 
E observed on the surface at $\left.\left(r[\mathrm{~km}], \phi I^{\circ}\right]\right)=(2,0)$

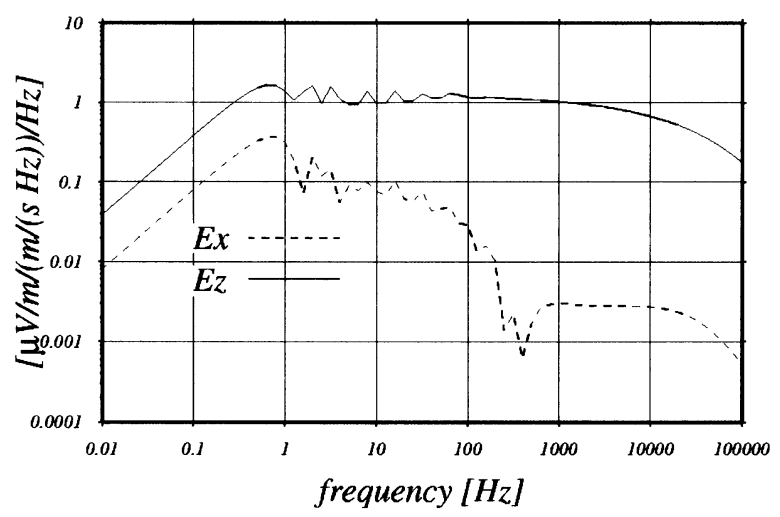

E observed on the surface at $\left.\left(r[\mathrm{~km}], \phi l^{\circ}\right]\right)=(2,0)$

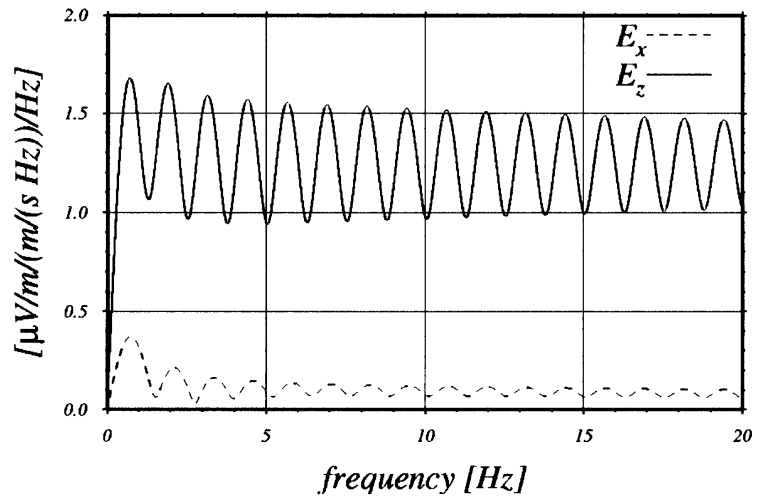

Fig. 2. The absolute value of complex Fourier components of the electric field. Results for the wide band range (upper) and the frequency range from $0[\mathrm{~Hz}]$ to $20[\mathrm{~Hz}]$ (lower)

\section{$E_{z}$ observed on the surface at $\left.\left(r[\mathrm{~km}], \phi I^{\circ}\right]\right)=(2,0)$}

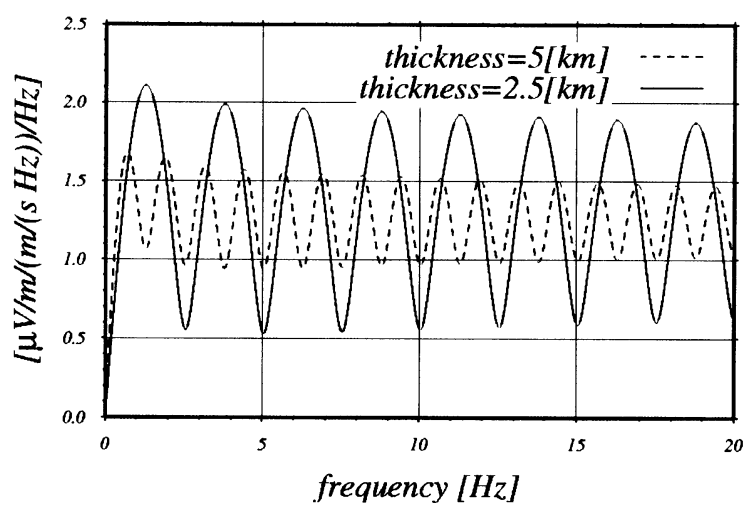

Fig. 3. Dependence of the resonant feature of the electric field on the thickness of the piezoelectric body.

where $\boldsymbol{v}$ is velocity of displacement and $\boldsymbol{B}_{0}$ is the ambient geomagnetic field. In order to ascertain effective observability of piezoelectric and motionally induced EM signals, we estimated the ratio $R$ between intensities of these two source
$E_{z}$ observed on the surface at $\left.\left(r[\mathrm{~km}], \phi I^{\circ}\right]\right)=(2,0)$

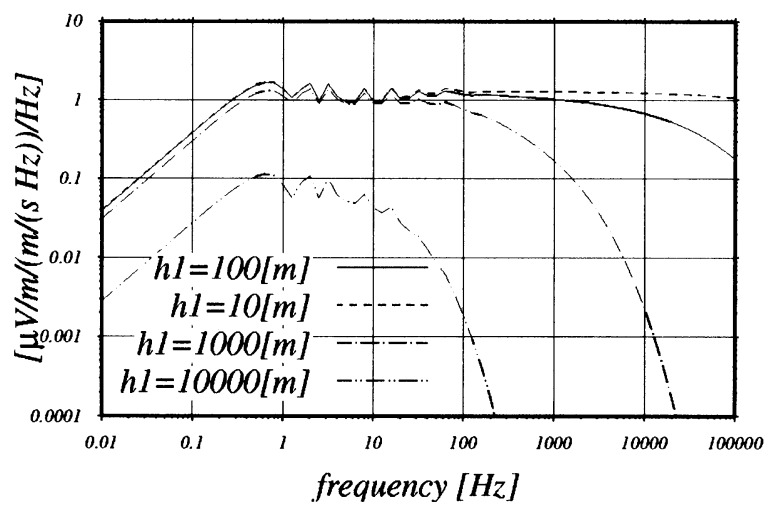

$E_{z}$ observed on the surface at $\left.\left(r[\mathrm{~km}], \phi l^{\circ}\right]\right)=(2,0)$

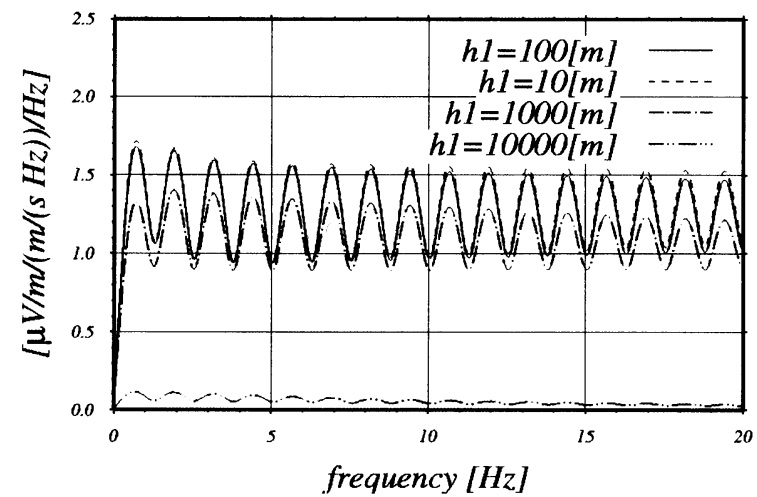

Fig. 4. $E_{z}$ with different $h_{1}$. Results for the wide band range (upper) and the frequency range from $0[\mathrm{~Hz}]$ to $20[\mathrm{~Hz}]$ (lower).

$E_{z}$ observed on the surface at $\left.\left(r[\mathrm{~km}], \phi I^{\circ}\right]\right)=(2,0)$

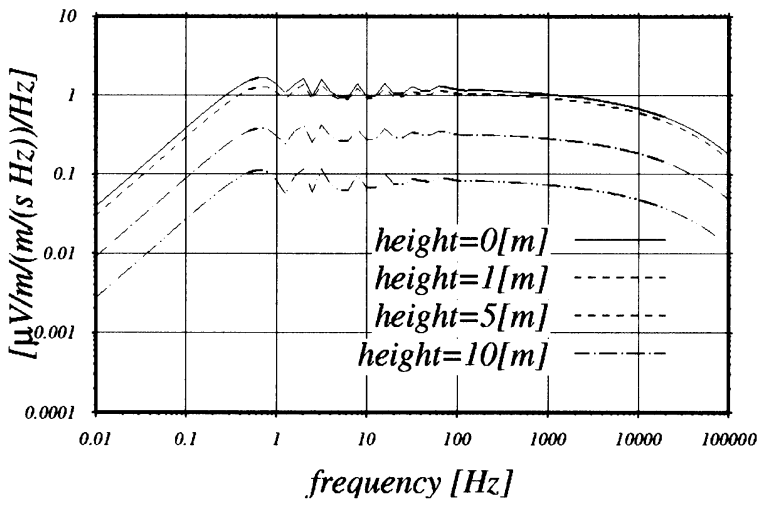

Fig. 5. Spatial variation of strength of $E_{z}$ with respect to the height.

components, $J_{\text {pe }}$ and $J_{\text {ind }}$ respectively. It is roughly given by

$$
R=\frac{J_{\mathrm{pe}}}{J_{\text {ind }}} \sim \frac{\left|d \frac{\partial T}{\partial t}\right|}{\left|\sigma v B_{0}\right|} \sim \frac{|d \rho \alpha \omega|}{\left|\sigma B_{0}\right|},
$$

where $d, T, v, B_{0}, \rho, \alpha$ and $\omega$ express the piezoelectric coefficients, the stress, the velocity, geomagnetic field intensity, the density, the speed and the angular frequency of in- 

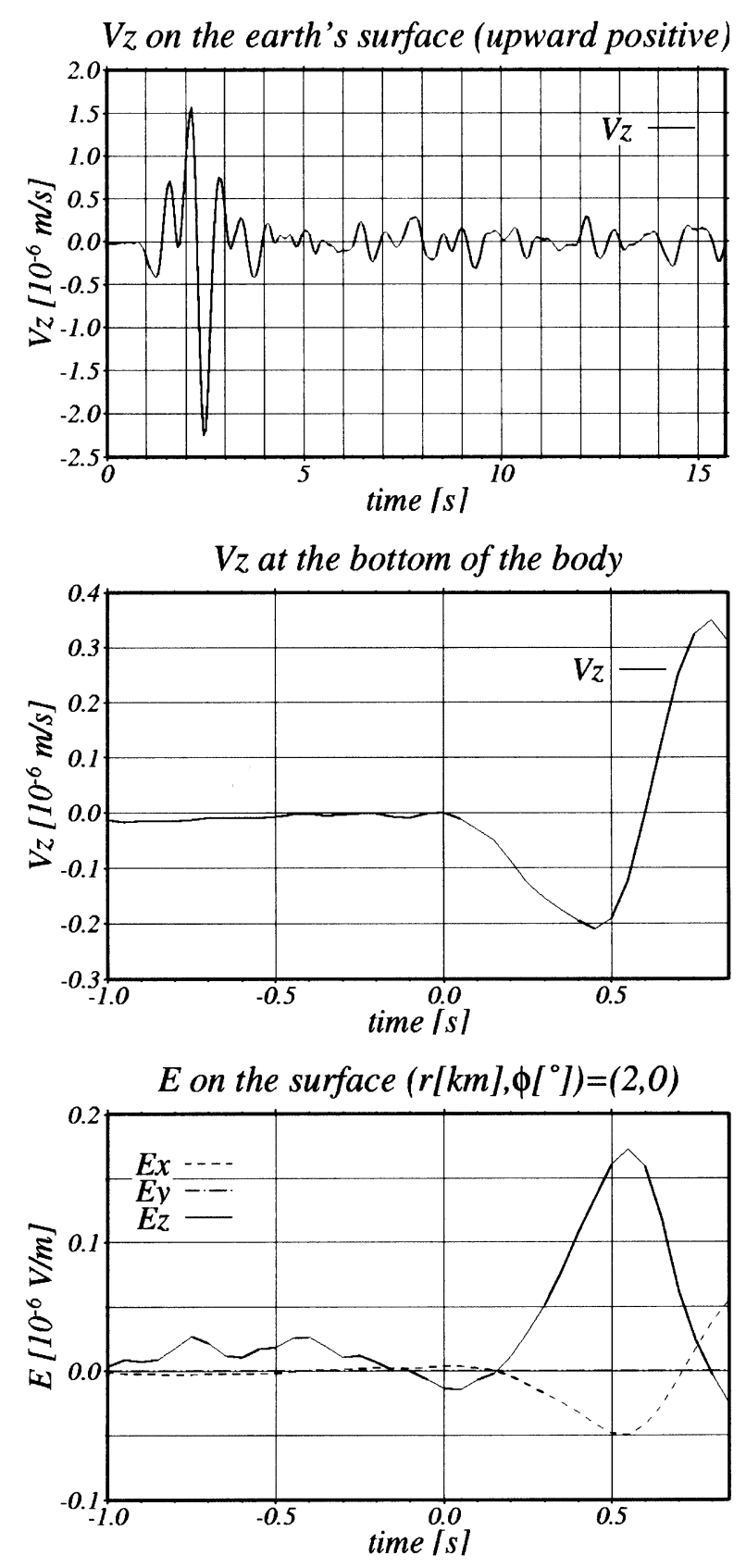

Fig. 6. The vertical component of incident seismic wave observed on the earth's surface (upper), that expected at the bottom of the piezoelectric body (center) and calculated 3 components of the electric field (lower). The origin of time is defined to express the arrival of incident $P$ wave at the bottom of the piezoelectric body.

cident $P$ wave, respectively. Substituting the parameters, $d \sim 1 \times 10^{-15}[\mathrm{C} / \mathrm{N}], \rho \sim 3 \times 10^{3}\left[\mathrm{~kg} / \mathrm{m}^{3}\right], \alpha \sim 6 \times 10^{3}$ $[\mathrm{m} / \mathrm{s}], \sigma \sim 1 \times 10^{-3}[\mathrm{~S} / \mathrm{m}]$ and $B_{0} \sim 3 \times 10^{-5}[\mathrm{~T}], R$ can be estimated as $R \sim 3 f$, where $f$ is the frequency. This estimation suggests that piezoelectric EM signals would be hidden and less detectable than the motionally induced signals in the frequency range lower than about $0.3 \mathrm{~Hz}$. At higher frequencies the piezoelectric field will dominate.

The electric field expected for an actual ground motion with the prescribed piezoelectric body is calculated and shown in Fig. 6. The upper trace in Fig. 6 is a vertical velocity record observed by a broad-band seismometer in- stalled at Tsukuba, Japan, which shows the PKPdf phase of the South Peru earthquake with $M_{W}=6.2$ on October 8 , 1998. The time origin was chosen when the incident PKPdf phase arrived at the bottom of the piezoelectric body. The center trace is the expected vertical velocity at the bottom of the piezoelectric body using the same model. Considering the boundary condition at the surface, the amplitude of $V_{z}$ at the bottom was assumed to be a half of that on the surface. The lower traces in Fig. 6 show three components of the electric field on the surface expected from the seismic record using the same model. The electric field after the arrival of the seismic wave at the surface is not shown because we used the whole space Green function for the elastic wave and therefore reflected waves cannot be considered in this study. It is shown by the present theory that the electric field arrives at the surface faster than the seismic wave. $E_{z}$ is large above the surface, but will be negligibly small just below the surface as has already been discussed. Above the surface, the noise level of $E_{z}$ is expected to be much higher than in the ground, and therefore detecting a coseismic signal is not easy, even if the piezoelectric coefficients are larger by 1 order in magnitude than those used in our present study.

In summary, this study indicated that the coseismic piezoelectric signal related to incidence of teleseismic body wave will be weak unless the ground motion is unusually strong. Therefore, it is not surprising that a coseismic change has rarely been observed by monitoring works of the electric field. On the other hand, the numerical calculation has shown that a wide band measurement, especially at frequencies higher than $1 \mathrm{~Hz}$, of the piezoelectric field may be useful in revealing the geometry of a buried piezoelectric body.

Acknowledgments. We would like to express our special thanks to M. J. S. Johnston for suggesting the present problem to us, and H. Shimizu for useful discussion. T. Urabe and F. Niu helped us to use the seismograph records. Calculations were made by the computer systems of the Earthquake Information Center of Earthquake Research Institute, University of Tokyo. We also wish to thank an anonymous reviewer and Dr. Don Russell for critical readings and helpful comments of the manuscript.

\section{Appendix}

Green tensors for EM fields in a half space, with an electric current element in another half space, can be derived and expressed as follows. EM fields at the location $\boldsymbol{r}=(x, y, z)$ due to an electric current element located in $\boldsymbol{r}^{\prime}=\left(x^{\prime}, y^{\prime}, z^{\prime}\right)$ are expressed. Symbols with a number 0 subscribed and those with a number 1 subscribed express those in the half space in which the fields are solved, and those in the half space in which the electric current element exists, respectively (see Fig. 1).

$$
\begin{gathered}
\mathbf{G}^{B}\left(\boldsymbol{r} \mid \boldsymbol{r}^{\prime} ; \omega\right)=\frac{\mu_{0}}{2 \pi}\left[\begin{array}{lll}
\gamma_{11}^{B} & \gamma_{12}^{B} & \gamma_{13}^{B} \\
\gamma_{21}^{B} & \gamma_{22}^{B} & \gamma_{23}^{B} \\
\gamma_{31}^{B} & \gamma_{22}^{B} & \gamma_{33}^{B}
\end{array}\right] \\
\gamma_{11}^{B}=\int_{0}^{\infty} d \lambda^{x} U_{0 z} e^{u_{0} z} \lambda \sum_{n=0}^{\infty} \epsilon_{n} J_{n}\left(\lambda r^{\prime}\right) \\
\times\left[\frac { - \lambda } { 4 } \left\{J_{n+2}(\lambda r) \sin (n+2) \phi\right.\right. \\
\left.\quad-J_{n-2}(\lambda r) \sin (n-2) \phi\right\} \cos n \phi^{\prime}
\end{gathered}
$$




$$
\begin{aligned}
& +\frac{\lambda}{4}\left\{J_{n+2}(\lambda r) \cos (n+2) \phi\right. \\
& \text { - } \left.\left.J_{n-2}(\lambda r) \cos (n-2) \phi\right\} \sin n \phi^{\prime}\right] \\
& \gamma_{21}^{B}=\int_{0}^{\infty} d \lambda^{x} U_{0 x} e^{u_{0} z} \lambda \sum_{n=0}^{\infty} \epsilon_{n} J_{n}\left(\lambda r^{\prime}\right) \\
& \times\left[u_{0} J_{n}(\lambda r) \cos (n) \phi \cos n \phi^{\prime}\right. \\
& \left.+u_{0} J_{n}(\lambda r) \sin (n) \phi \sin n \phi^{\prime}\right] \\
& +\int_{0}^{\infty} d \lambda^{x} U_{0 z} e^{u_{0} z} \lambda \sum_{n=0}^{\infty} \epsilon_{n} J_{n}\left(\lambda r^{\prime}\right) \\
& \times\left[\frac { \lambda } { 4 } \left\{J_{n+2}(\lambda r) \cos (n+2) \phi\right.\right. \\
& -2 J_{n}(\lambda r) \cos (n) \phi \\
& \left.+J_{n-2}(\lambda r) \cos (n-2) \phi\right\} \cos n \phi^{\prime} \\
& +\frac{\lambda}{4}\left\{J_{n+2}(\lambda r) \sin (n+2) \phi\right. \\
& -2 J_{n}(\lambda r) \sin (n) \phi \\
& \left.\left.+J_{n-2}(\lambda r) \sin (n-2) \phi\right\} \sin n \phi^{\prime}\right] \\
& \gamma_{31}^{B}=\int_{0}^{\infty} d \lambda^{x} U_{0 x} e^{u_{0} z} \lambda \sum_{n=0}^{\infty} \epsilon_{n} J_{n}\left(\lambda r^{\prime}\right) \\
& \times\left[\frac { \lambda } { 2 } \left\{J_{n+1}(\lambda r) \sin (n+1) \phi\right.\right. \\
& \left.+J_{n-1}(\lambda r) \sin (n-1) \phi\right\} \cos n \phi^{\prime} \\
& -\frac{\lambda}{2}\left\{J_{n+1}(\lambda r) \cos (n+1) \phi\right. \\
& \left.\left.+J_{n-1}(\lambda r) \cos (n-1) \phi\right\} \sin n \phi^{\prime}\right] \\
& \gamma_{12}^{B}=\int_{0}^{\infty} d \lambda^{x} U_{0 z} e^{u_{0} z} \lambda \sum_{n=0}^{\infty} \epsilon_{n} J_{n}\left(\lambda r^{\prime}\right) \\
& \times\left[\frac { \lambda } { 4 } \left\{J_{n+2}(\lambda r) \cos (n+2) \phi\right.\right. \\
& +2 J_{n}(\lambda r) \cos (n) \phi \\
& \left.+J_{n-2}(\lambda r) \cos (n-2) \phi\right\} \cos n \phi^{\prime} \\
& +\frac{\lambda}{4}\left\{J_{n+2}(\lambda r) \sin (n+2) \phi\right. \\
& +2 J_{n}(\lambda r) \sin (n) \phi \\
& \left.\left.+J_{n-2}(\lambda r) \sin (n-2) \phi\right\} \sin n \phi^{\prime}\right] \\
& -\int_{0}^{\infty} d \lambda^{x} U_{0 x} e^{u_{0} z} \lambda \sum_{n=0}^{\infty} \epsilon_{n} J_{n}\left(\lambda r^{\prime}\right) \\
& \times\left[u_{0} J_{n}(\lambda r) \cos (n) \phi \cos n \phi^{\prime}\right. \\
& \left.+u_{0} J_{n}(\lambda r) \sin (n) \phi \sin n \phi^{\prime}\right] \\
& \gamma_{22}^{B}=-\gamma_{11}^{B} \\
& \gamma_{32}^{B}=\int_{0}^{\infty} d \lambda^{x} U_{0 x} e^{u_{0} z} \lambda \sum_{n=0}^{\infty} \epsilon_{n} J_{n}\left(\lambda r^{\prime}\right) \\
& \times\left[\frac { - \lambda } { 2 } \left\{J_{n+1}(\lambda r) \cos (n+1) \phi\right.\right. \\
& \text { - } \left.J_{n-1}(\lambda r) \cos (n-1) \phi\right\} \cos n \phi^{\prime} \\
& +\frac{-\lambda}{2}\left\{J_{n+1}(\lambda r) \sin (n+1) \phi\right. \\
& \left.\left.-J_{n-1}(\lambda r) \sin (n-1) \phi\right\} \sin n \phi^{\prime}\right]
\end{aligned}
$$$$
\gamma_{13}^{B}=\int_{0}^{\infty} d \lambda^{z} U_{0 z} e^{u_{0} z} \lambda \sum_{n=0}^{\infty} \epsilon_{n} J_{n}\left(\lambda r^{\prime}\right)
$$$$
\times\left[\frac { - \lambda } { 2 } \left\{J_{n+1}(\lambda r) \sin (n+1) \phi\right.\right.
$$$$
\left.+J_{n-1}(\lambda r) \sin (n-1) \phi\right\} \cos n \phi^{\prime}
$$$$
+\frac{\lambda}{2}\left\{J_{n+1}(\lambda r) \cos (n+1) \phi\right.
$$$$
\left.\left.+J_{n-1}(\lambda r) \cos (n-1) \phi\right\} \sin n \phi^{\prime}\right]
$$$$
\gamma_{23}^{B}=\int_{0}^{\infty} d \lambda^{z} U_{0 z} e^{u_{0} z} \lambda \sum_{n=0}^{\infty} \epsilon_{n} J_{n}\left(\lambda r^{\prime}\right)
$$$$
\times\left[\frac { \lambda } { 2 } \left\{J_{n+1}(\lambda r) \cos (n+1) \phi\right.\right.
$$$$
\left.-J_{n-1}(\lambda r) \cos (n-1) \phi\right\} \cos n \phi^{\prime}
$$$$
+\frac{\lambda}{2}\left\{J_{n+1}(\lambda r) \sin (n+1) \phi\right.
$$$$
\left.\left.-J_{n-1}(\lambda r) \sin (n-1) \phi\right\} \sin n \phi^{\prime}\right]
$$$$
\gamma_{33}^{B}=0
$$$$
\mathbf{G}^{E}\left(\boldsymbol{r} \mid \boldsymbol{r}^{\prime} ; \omega\right)=\frac{1}{2 \pi \sigma_{0}^{\prime}}\left[\begin{array}{lll}
\gamma_{11}^{E} & \gamma_{12}^{E} & \gamma_{13}^{E} \\
\gamma_{21}^{E} & \gamma_{22}^{E} & \gamma_{23}^{E} \\
\gamma_{31}^{E} & \gamma_{22}^{E} & \gamma_{33}^{E}
\end{array}\right]
$$$$
\gamma_{11}^{E}=\int_{0}^{\infty} d \lambda \frac{1}{2}\left(\lambda^{x} U_{0 x}-u_{0}{ }^{x} U_{0 z}\right)
$$$$
\times e^{u_{0} z} \lambda \sum_{n=0}^{\infty} \epsilon_{n} J_{n}\left(\lambda r^{\prime}\right)\left[\frac { \lambda } { 2 } \left\{J_{n+2}(\lambda r) \cos (n+2) \phi\right.\right.
$$$$
\left.+J_{n-2}(\lambda r) \cos (n-2) \phi\right\} \cos n \phi^{\prime}
$$$$
+\frac{\lambda}{2}\left\{J_{n+2}(\lambda r) \sin (n+2) \phi\right.
$$$$
\left.\left.+J_{n-2}(\lambda r) \sin (n-2) \phi\right\} \sin n \phi^{\prime}\right]
$$$$
+\int_{0}^{\infty} d \lambda\left(\frac{\lambda}{2} u_{0}^{x} U_{0 z}-\left(\frac{\lambda^{2}}{2}+\gamma_{0}^{2}\right){ }^{x} U_{0 x}\right)
$$$$
\times e^{u_{0} z} \lambda \sum_{n=0}^{\infty} \epsilon_{n} J_{n}\left(\lambda r^{\prime}\right)\left[J_{n}(\lambda r) \cos (n) \phi \cos n \phi^{\prime}\right.
$$$$
\left.+J_{n}(\lambda r) \sin (n) \phi \sin n \phi^{\prime}\right]
$$$$
\gamma_{21}^{E}=\int_{0}^{\infty} d \lambda \frac{1}{2}\left(\lambda^{x} U_{0 x}-u_{0}{ }^{x} U_{0 z}\right)
$$$$
\times e^{u_{0} z} \lambda \sum_{n=0}^{\infty} \epsilon_{n} J_{n}\left(\lambda r^{\prime}\right)\left[\frac { \lambda } { 2 } \left\{J_{n+2}(\lambda r) \sin (n+2) \phi\right.\right.
$$$$
\text { - } \left.J_{n-2}(\lambda r) \sin (n-2) \phi\right\} \cos n \phi^{\prime}
$$$$
-\frac{\lambda}{2}\left\{J_{n+2}(\lambda r) \cos (n+2) \phi\right.
$$

$$
\begin{gathered}
\left.\left.-J_{n-2}(\lambda r) \cos (n-2) \phi\right\} \sin n \phi^{\prime}\right] \\
\gamma_{31}^{E}=\int_{0}^{\infty} d \lambda\left(\lambda^{x} U_{0 z}-u_{0}{ }^{x} U_{0 x}\right) \\
\times e^{u_{0} z} \lambda \sum_{n=0}^{\infty} \epsilon_{n} J_{n}\left(\lambda r^{\prime}\right)\left[\frac { \lambda } { 2 } \left\{J_{n+1}(\lambda r) \cos (n+1) \phi\right.\right. \\
\left.-J_{n-1}(\lambda r) \cos (n-1) \phi\right\} \cos n \phi^{\prime} \\
+\frac{\lambda}{2}\left\{J_{n+1}(\lambda r) \sin (n+1) \phi\right.
\end{gathered}
$$




$$
\begin{array}{rlrl}
\left.\left.-J_{n-1}(\lambda r) \sin (n-1) \phi\right\} \sin n \phi^{\prime}\right] & (\text { A.14) } & { }^{x} U_{0 x} & =\frac{2 N_{0}}{N_{1}+N_{0}} \frac{1}{u_{0}} e^{-u_{1} z^{\prime}}, \\
\gamma_{12}^{E}=\gamma_{21}^{E} & (\mathrm{~A} .15) & { }^{x} U_{0 z} & =\frac{2\left(K_{0} N_{0}-K_{1} N_{1}\right)}{\left(K_{1}+K_{0}\right)\left(N_{1}+N_{0}\right)} \frac{1}{\lambda} e^{-u_{1} z^{\prime}} \\
\gamma_{22}^{E}=\int_{0}^{\infty} d \lambda \frac{1}{2}\left(u_{0}{ }^{x} U_{0 z}-\lambda^{x} U_{0 x}\right) & { }^{z} U_{0 z} & =\frac{2 K_{1}}{K_{1}+K_{0}} \frac{1}{u_{1}} e^{-u_{1} z^{\prime}}
\end{array}
$$$$
\times e^{u_{0} z} \lambda \sum_{n=0}^{\infty} \epsilon_{n} J_{n}\left(\lambda r^{\prime}\right)\left[\frac { \lambda } { 2 } \left\{J_{n+2}(\lambda r) \cos (n+2) \phi\right.\right.
$$$$
\left.+J_{n-2}(\lambda r) \cos (n-2) \phi\right\} \cos n \phi^{\prime}
$$$$
+\frac{\lambda}{2}\left\{J_{n+2}(\lambda r) \sin (n+2) \phi\right.
$$$$
\left.\left.+J_{n-2}(\lambda r) \sin (n-2) \phi\right\} \sin n \phi^{\prime}\right]
$$$$
+\int_{0}^{\infty} d \lambda\left(\frac{\lambda}{2} u_{0}^{x} U_{0 z}-\left(\frac{\lambda^{2}}{2}+\gamma_{0}^{2}\right){ }^{x} U_{0 x}\right)
$$$$
\times e^{u_{0} z} \lambda \sum_{n=0}^{\infty} \epsilon_{n} J_{n}\left(\lambda r^{\prime}\right)\left[J_{n}(\lambda r) \cos (n) \phi \cos n \phi^{\prime}\right.
$$$$
\left.+J_{n}(\lambda r) \sin (n) \phi \sin n \phi^{\prime}\right]
$$$$
\gamma_{32}^{E}=\int_{0}^{\infty} d \lambda\left(\lambda^{x} U_{0 z}-u_{0}^{x} U_{0 x}\right)
$$$$
\times e^{u_{0} z} \lambda \sum_{n=0}^{\infty} \epsilon_{n} J_{n}\left(\lambda r^{\prime}\right)\left[\frac { \lambda } { 2 } \left\{J_{n+1}(\lambda r) \sin (n+1) \phi\right.\right.
$$$$
\left.+J_{n-1}(\lambda r) \sin (n-1) \phi\right\} \cos n \phi^{\prime}
$$$$
-\frac{\lambda}{2}\left\{J_{n+1}(\lambda r) \cos (n+1) \phi\right.
$$$$
\left.\left.+J_{n-1}(\lambda r) \cos (n-1) \phi\right\} \sin n \phi^{\prime}\right]
$$$$
\gamma_{13}^{E}=\int_{0}^{\infty} d \lambda u_{0}^{z} U_{0 z}
$$$$
\times e^{u_{0} z} \lambda \sum_{n=0}^{\infty} \epsilon_{n} J_{n}(\lambda r)\left[\frac { - \lambda } { 2 } \left\{J_{n+1}(\lambda r) \cos (n+1) \phi\right.\right.
$$$$
\left.-J_{n-1}(\lambda r) \cos (n-1) \phi\right\} \cos n \phi^{\prime}
$$$$
+\frac{-\lambda}{2}\left\{J_{n+1}(\lambda r) \sin (n+1) \phi\right.
$$$$
\left.\left.-J_{n-1}(\lambda r) \sin (n-1) \phi\right\} \sin n \phi^{\prime}\right]
$$$$
\gamma_{23}^{E}=\int_{0}^{\infty} d \lambda u_{0}{ }^{z} U_{0 z}
$$$$
\times e^{u_{0} z} \lambda \sum_{n=0}^{\infty} \epsilon_{n} J_{n}\left(\lambda r^{\prime}\right)\left[\frac { - \lambda } { 2 } \left\{J_{n+1}(\lambda r) \sin (n+1) \phi\right.\right.
$$$$
\left.+J_{n-1}(\lambda r) \sin (n-1) \phi\right\} \cos n \phi^{\prime}
$$$$
-\frac{-\lambda}{2}\left\{J_{n+1}(\lambda r) \cos (n+1) \phi\right.
$$$$
\left.\left.+J_{n-1}(\lambda r) \cos (n-1) \phi\right\} \sin n \phi^{\prime}\right]
$$$$
\gamma_{33}^{E}=\int_{0}^{\infty} d \lambda u_{0}^{z} U_{0 z}
$$$$
\times e^{u_{0} z} \lambda \sum_{n=0}^{\infty} \epsilon_{n} J_{n}\left(\lambda r^{\prime}\right)\left[\lambda J_{n}(\lambda r) \cos (n) \phi \cos n \phi^{\prime}\right.
$$$$
\left.+\lambda J_{n}(\lambda r) \sin (n) \phi \sin n \phi^{\prime}\right]
$$

Some symbols in the expressions above are defined as follows.

$$
R=\sqrt{\left(x-x^{\prime}\right)^{2}+\left(y-y^{\prime}\right)^{2}},
$$

and for $j=0$ and 1 ,

$$
\begin{gathered}
N_{j}=\frac{u_{j}}{-i \omega \mu_{j}}, \quad K_{j}=\frac{u_{j}}{\sigma_{j}^{\prime}}, \\
\sigma_{j}^{\prime}=\sigma_{j}-i \omega \varepsilon_{j}, \quad \gamma_{j}^{2}=-i \omega \mu_{j} \sigma_{j}^{\prime}, \\
u_{j}=\sqrt{\lambda^{2}+\gamma_{j}^{2}} .
\end{gathered}
$$

For convenience, the addition theorem for the Bessel functions as follows (Watson, 1944) was applied,

$$
\begin{aligned}
& J_{0}(\lambda R) \\
&= J_{0}\left(\lambda \sqrt{r^{2}+r^{\prime 2}-2 r r^{\prime} \cos \left(\phi-\phi^{\prime}\right)}\right) \\
&= \sum_{n=0}^{\infty} \epsilon_{n} J_{n}(\lambda r) J_{n}\left(\lambda r^{\prime}\right) \cos n\left(\phi-\phi^{\prime}\right), \\
& \epsilon_{n}=\left\{\begin{array}{ll}
1 & n=0 \\
2 & n>0
\end{array} .\right.
\end{aligned}
$$

In this study, the conditions $\mu_{0}=\mu_{1}, \sigma_{0} \rightarrow 0$ and $\varepsilon_{0}, \varepsilon_{1} \rightarrow 0$ are assumed.

\section{References}

Aki, K. and P. G. Richards, Quantitative Seismology, Theory and Methods, Vol. 1, 557pp., W. H. Freeman and company, New York, 1980.

Anderson, W. L., Fast Hankel transforms using related and lagged convolutions, ACM Trans. Math. Softw., 8, 344-368, 1982.

Bishop, J. R., Estimating quartz fabrics from piezoelectric measurements, Math. Geol., 13, 261-289, 1981a.

Bishop, J. R., Piezoelectric effects in quartz-rich rocks, Tectonophys., 77, 297-321, $1981 b$.

Eleman, F., The response of magnetic instrument to earthquake waves, $J$ Geomag. Geoelectr., 18, 43-72, 1965.

Fraser-Smith, A. C., A. Bernardi, P. R. McGill, M. E. Ladd, R. A. Heliwell, and O. G. Villard, Jr., Low-frequency magnetic field measurements near the epicenter of the $M_{s} 7.1$ Loma Prieta earthquake, Geophys. Res. Lett. 17, 1465-1468, 1990 .

Ghomshei, M. M. and T. L. Templeton, Piezoelectric and $a$-axes fabric along a quartz vein, Phys. Earth Planet. Inter., 55, 374-386, 1989.

Ghomshei, M. M., B. B. Narod, T. L. Templeton, A. S. Arrott, and R. D. Russell, Piezoelectric pole figure of a vein quartz sample, Text. Microstruct. 7, 303-316, 1988.

Gokhberg, M. B., V. A. Morgounov, T. Yoshino, and I. Tomizawa, Experimental measurement of electromagnetic emissions possibly related to earthquakes in Japan, J. Geophys. Res., 87, 7824-7828, 1982.

Huang, Q., Theoretical and experimental study on seismoelectric signals and earthquake-related phenomena, $\mathrm{Ph}$. D. thesis, Osaka University, 107 pp., 1999.

Ikeda, T., Fundamentals of Piezoelectricity, 263 pp., Oxford university press, Oxford, 1990.

Johnston, M. J. S., R. J. Mueller, and Y. Sasai, Magnetic field observation in the near-field the 28 June $1992 M_{w} 7.3$ Landers, California, earthquake, Bull. Seis. Soc. Am., 84, 792-798, 1994.

Kepic, A. W., M. Maxwell, and R. D. Russell, Field trials of a seismoelectric method for detecting massive sulfides, Geophys., 60, 365-373, 1995.

Mikhailov, O. V., M. W. Haartsen, and M. N. Toksöz, Electroseismic investigation of the shallow subsurface: Field measurements and numerical modeling, Geophys., 62, 97-105, 1997.

Mueller, R. J. and M. J. S. Johnston, Seismomagnetic effect generated by the October 18, 1989, $M_{l}$ 7.1 Loma Prieta, California, Earthquake, Geophys. 
Res. Lett., 17, 1231-1234, 1990.

Ogawa, T. and H. Utada, Coseismic piezoelectric effects due to a dislocation 1: An analytic far and early-time field solution in a homogeneous whole space., Phys. Earth Planet. Inter, 2000 (submitted)

Parkhomenko, E. I., Electrification Phenomena in Rocks, 314 pp., Plenum Press, New York, 1971 (Translated from Russian by George V. Keller).

Russell, R. D. and A. S. J. Barker, Seismo-electric exploration: expected signal amplitudes, Geophys. Prospect., 39, 105-118, 1991.

Sasai, Y., Tectonomagnetic modeling on the basis of the linear piezomagnetic effect, Bull. Earthq. Res. Inst., 66, 585-722, 1991.

Stoyer, C. H., Electromagnetic fields of dipoles in stratified media, IEEE Trans. Ant. Propag., 25, 547-552, 1977.

Tuck, G. J., F. D. Stacey, and J. Starkey, A search for the piezoelectric effect in quartz-bearing rocks, Tectonophys., 39, T7-T11, 1977.
Warwick, J. W., C. Stoker, and T. R. Meyer, Radio emission associated with rock fracture: Possible application to the great Chilean earthquake of May 22, 1960, J. Geophys. Res., 87, 2851-2859, 1982.

Watson, G. N., A Treatise on the Theory of Bessel Functions, second edition, 804 pp., Cambridge University Press, London, 1944.

Yoshida, S., P. Manjgaladze, D. Zilpimiani, M. Ohnaka, and M. Nakatani, Electromagnetic emissions associated with frictional sliding of rock, in Electromagnetic Phenomena Related to Earthquake Prediction, edited by M. Hayakawa and Y. Fujinawa, pp. 307-322, Terrapub, Tokyo, 1994. Yoshii, T., Crustal Structure in Japan, 121 pp., University of Tokyo Press, Tokyo, 1979 (in Japanese).

T. Ogawa (email: ogawa@eri.u-tokyo.ac.jp) and H. Utada (e-mail: utada@eri.u-tokyo.ac.jp) 